\title{
Insider Trading Equilibrium in a Market with Memory
}

\author{
Francesca Biagini ${ }^{1), 3)}$, Yaozhong $\mathrm{Hu}^{2), 3)}$, Thilo Meyer-Brandis ${ }^{1)}$ \\ and Bernt Øksendal ${ }^{3), 4)}$
}

30 August 2011

\begin{abstract}
We consider the Kyle-Back model for insider trading, with the difference that the classical Brownian motion noise of the noise traders is replaced by the noise of a fractional Brownian motion $B^{H}$ with Hurst parameter $H>\frac{1}{2}$ (when $H=\frac{1}{2}, B^{H}$ coincides with the classical Brownian motion). Heuristically, for $H>\frac{1}{2}$ this means that the noise traders has some "memory", in the sense that any increment from time $t$ on has a positive correlation with its value at $t$. (In other words, the noise trading is a persistent stochastic process). It also means that the paths of the noise trading process are more regular than in the classical Brownian motion case.

We obtain an equation for the optimal (relative) trading intensity for the insider in this setting, and we show that when $H \rightarrow \frac{1}{2}$ the solution converges to the solution in the classical case. Finally, we discuss how the size of the Hurst coefficient $H$ influences the optimal performance and portfolio of the insider.
\end{abstract}

\section{Introduction}

In their seminal papers Kyle [6] and subsequently Back [2] formulate and study an equilibrium model for insider trading. There are many papers followed Kyle-Back inspired models that should be cited. The paper most closely related to ours in setup and method is [1], where a (classical) Brownian motion model is studied. Here we review then briefly the Kyle-Back model, based on the presentation in [1]. We assume the financial market has three agents:

- (i) The insider, who already from the initial time $t=0$ knows the value $\tilde{v}$ at the terminal time $t=T$ of a given stock. The portfolio of the insider, measured in terms of the number of stocks held at time $t$, is denoted by $x_{t}, t \in[0, T]$. It is assumed that $\tilde{v}$ is a centered Gaussian random variable of known variance.

\footnotetext{
${ }^{0}$ Mathematics Subject Classifications (2010). Primary 60G22, 91G80; Secondary 60G35.

Key words and phrases: insider trading, memory, fractional Brownian motion, filtering problem, optimal expected wealth.

The research leading to these results has received funding from the European Research Council under the European Community's Seventh Framework Programme (FP7/2007-2013) / ERC grant agreement no [228087]. $\mathrm{Y}$. Hu is also partially supported by a grant from the Simons Foundation \#209206.
} 
- (ii) The noise traders, who trade randomly without any information about the market. The portfolio $z_{t}$ of the noise traders is assumed to have the form

$$
d z_{t}=\sigma_{t} d B_{t}, \quad t \in[0, T],
$$

where $\sigma_{t}$ is a given continuous deterministic function and $B_{t}=B_{t}(\omega),(t, \omega) \in[0, T] \times \Omega$, is a Brownian motion on a filtered probability space $\left(\Omega, \mathcal{F},\left\{\mathcal{F}_{t}\right\}_{t \in[0, T]}, \mathbb{P}\right)$. It is assumed that $\tilde{v}$ is independent of the Brownian motion $B_{t}, t \in[0, T]$.

- (ii) The market makers, who at any time $t$ can observe the total traded volume

$$
y_{t}=x_{t}+z_{t},
$$

but not the separate trades $x_{t}, z_{t}$. Based on the information (filtration) $\mathcal{F}_{t}^{y}, t \in[0, T]$, generated by the observations $y_{s}, s \leq t$, the market makers set the price of the stock at time $t$ equal to

$$
p_{t}:=\mathbb{E}\left[\tilde{v} \mid \mathcal{F}_{t}^{y}\right], \quad 0 \leq t \leq T
$$

The wealth $w_{t}$ at time $t$ of the insider can be expressed as

$$
w_{t}=w_{0}+\int_{0}^{t} x_{s} d p_{s}, \quad 0 \leq t \leq T
$$

A priori this is an anticipative stochastic integral, which needs further explanation. If we assume, as Kyle and Back, that the strategy of the insider has the form

$$
d x_{t}=\left(\tilde{v}-p_{t}\right) \beta_{t} d t
$$

for some deterministic continuous function $\beta_{t}>0$, called the insider trading intensity, then a natural interpretation of (1.4) is obtained by using integration by parts, as follows:

$$
\begin{aligned}
w_{t} & =w_{0}+x_{t} p_{t}-\int_{0}^{t} p_{s} d x_{s} \\
& =w_{0}+p_{t} \int_{0}^{t}\left(\tilde{v}-p_{s}\right) \beta_{s} d s-\int_{0}^{t} p_{s}\left(\tilde{v}-p_{s}\right) \beta_{s} d s \\
& =w_{0}+\int_{0}^{t}\left(\tilde{v}-p_{s}\right)^{2} \beta_{s} d s-\int_{0}^{t}\left(\tilde{v}-p_{t}\right)\left(\tilde{v}-p_{s}\right) \beta_{s} d s
\end{aligned}
$$


Alternatively, one might obtain (1.6) by interpreting the stochastic integral in (1.4) as a forward integral. See [8] for definitions and [1] for applications of forward integrals to finance. The insider tries to find the trading intensity $\beta_{t}$ which maximizes the expected terminal wealth

$$
\mathbb{E}\left[w_{T}\right]=\mathbb{E}\left[w_{T}^{(\beta)}\right]=w_{0}+\int_{0}^{T} \mathbb{E}\left[\left(\tilde{v}-p_{s}\right)^{2}\right] \beta_{s} d s-\int_{0}^{T} \mathbb{E}\left[\left(\tilde{v}-p_{T}\right)\left(\tilde{v}-p_{s}\right)\right] \beta_{s} d s .
$$

The dilemma for the insider is that an increased trading intensity at some time $t$ will reveal more information about the value of $\tilde{v}$ to the market makers and hence induce a price $p_{t}$ closer to $\tilde{v}$, which in turn implies a reduced insider advantage. The optimal insider trading strategy is proved to be

$$
\beta_{t}=\frac{\sigma_{t}^{2}\left(\int_{0}^{T} \sigma_{s}^{2} d s\right)^{\frac{1}{2}}}{S_{0}^{\frac{1}{2}} \int_{t}^{T} \sigma_{s}^{2} d s} ; \quad S_{0}=\mathbb{E}\left[(\tilde{v}-\mathbb{E}[\tilde{v}])^{2}\right],
$$

which gives the optimal mean square error

$$
S_{t}=\mathbb{E}\left[\left(\tilde{v}-p_{t}\right)^{2}\right]=\frac{S_{0} \int_{t}^{T} \sigma_{s}^{2} d s}{\int_{0}^{T} \sigma_{s}^{2} d s},
$$

and the optimal insider performance

$$
J(\beta):=\mathbb{E}\left[w_{T}^{(\beta)}\right]=w_{0}+\left(S_{0} \int_{0}^{T} \sigma_{s}^{2} d s\right)^{\frac{1}{2}} .
$$

In particular, this implies $p_{T}=\tilde{v}$ and $p_{t}=\mathbb{E}[\tilde{v}]+\lambda y_{t}$, where $\lambda=\left(\frac{S_{0}}{\int_{0}^{T} \sigma_{s}^{2} d s}\right)^{\frac{1}{2}}$ is called the price sensitivity. See [1] for details.

The purpose of this paper is to study the above model in the case when the Brownian motion $B$ in (1.1) is generalized to a fractional Brownian motion $B^{H}$ with Hurst parameter $H \in(0,1)$. By definition $B_{t}^{H}, 0 \leq t \leq T$, is a continuous and centered Gaussian process with covariance function

$$
\mathbb{E}\left[B_{t}^{H} B_{s}^{H}\right]=\frac{1}{2}\left(t^{2 H}+s^{2 H}-|t-s|^{2 H}\right), \quad 0 \leq t \leq T .
$$

If $H=\frac{1}{2}$ then $B^{H}$ is the classical Brownian motion $B$. If $H \neq \frac{1}{2}$ the increments of $B^{H}$ are not independent. For $H>\frac{1}{2}$ the two increments

$$
B_{t+h}^{H}-B_{t}^{H} \quad \text { and } \quad B_{s+h}^{H}-B_{s}^{H}
$$


are positively correlated, while they are negatively correlated for $H<\frac{1}{2}$. Thus the case for $H>\frac{1}{2}$ corresponds to systems with memory and persistence, while the case of $H<\frac{1}{2}$ corresponds to systems with turbulence and anti-persistence. If $H>\frac{1}{2}$ then the paths of $B^{H}$ are more regular than for classical Brownian motion, while if $H<\frac{1}{2}$ the paths are less regular. More precisely, for any $\alpha<H$ the paths of $B^{H}$ are Hölder continuous with exponent $\alpha$ almost surely, i.e.

$$
\left|B_{t}^{H}-B_{s}^{H}\right| \leq c|t-s|^{\alpha}, \quad 0 \leq t \leq T
$$

for some (random) constant $c>0$. For more information about fractional Brownian motion and its applications, we refer to [3], [7] and the references therein.

In this paper we restrict ourselves to the case $H>\frac{1}{2}$. In other words, we study how the introduction of persistence or memory among the noise traders influences the KyleBack model, in particular what effect it has on the optimal insider portfolio and maximal expected insider wealth. As in the Kyle-Back setting, we assume that $\tilde{v}$ is independent of $B_{t}^{H}, t \in[0, T]$. We prove that if an optimal smooth insider trading intensity $\beta$ exists, then it is the solution of a non-linear integro-differential equation. Moreover, we show that $p_{T}=\tilde{v}$ in Theorem 2.4.

In the formulation adopted in this paper, we have encountered a new stochastic differential equations

$$
d y_{t}=\left(\tilde{v}-\mathbb{E}\left(\tilde{v} \mid \mathcal{F}_{t}^{y}\right) d t+\sigma_{t} d B_{t}^{H} .\right.
$$

The existence and uniqueness of the solution to the above equation have not been studied yet. In Section 2 we shall formulate our problem, obtain an existence result for the above equation by using the innovation technique, and find an equation that the maximum trading intensity must satisfy. In Section 3, we study the uniqueness of the above equation. In Section 4, we discuss the impact of long memory on the insider trader. The Appendix provide some technical results.

\section{The main result}

We use the same setup as in Section 1 , except that $B_{t}, 0 \leq t \leq T$, is replaced with a fractional Brownian motion $B_{t}^{H}, 0 \leq t \leq T$, with Hurst parameter $H>\frac{1}{2}$. Thus the portfolio of the noise traders gets the form

$$
d z_{t}=\sigma_{t} d B_{t}^{H}, \quad t \in[0, T]
$$

the portfolio of the insider is as before

$$
d x_{t}=\left(\tilde{v}-p_{t}\right) \beta_{t} d t
$$

where $p_{t}$ is the market price at time $t$ set by the market makers, which will be made more precise in next lines (see equation (2.4) below). The total traded volume is hence 


$$
d y_{t}=\left(\tilde{v}-p_{t}\right) \beta_{t} d t+\sigma_{t} d B_{t}^{H}
$$

If we let $\mathcal{F}_{t}^{y}, t \in[0, T]$, be the filtration generated by $y_{s}, s \leq t$, then it is assumed that

$$
p_{t}:=\mathbb{E}\left[\tilde{v} \mid \mathcal{F}_{t}^{y}\right], \quad 0 \leq t \leq T .
$$

Substituting this into (2.3) we get that the total traded volume process must satisfy the equation

$$
d y_{t}=\left(\tilde{v}-\mathbb{E}\left[\tilde{v} \mid \mathcal{F}_{t}^{y}\right]\right) \beta_{t} d t+\sigma_{t} d B_{t}^{H}, \quad t \in[0, T] .
$$

As in [1] we will prove that it is possible to find a solution of (2.5) by regarding $y_{t}, 0 \leq t \leq T$, as the innovation process $\tilde{y}_{t}, 0 \leq t \leq T$, of an auxiliary linear filtering problem, where the signal process is

$$
\xi_{t}=\tilde{v} ; \quad t \in[0, T]
$$

and the observation process is

$$
d \hat{y}_{t}=\tilde{v} \beta_{t} d t+\sigma_{t} d B_{t}^{H} ; \quad t \in[0, T], \hat{y}_{0}=0 .
$$

The innovation process for this problem is, by definition,

$$
\begin{aligned}
d \tilde{y}_{t} & =\left(\tilde{v}-\mathbb{E}\left[\tilde{v} \mid \mathcal{F}_{t}^{\hat{y}}\right]\right) \beta_{t} d t+\sigma_{t} d B_{t}^{H} \\
& =d \hat{y}_{t}-\mathbb{E}\left[\tilde{v} \mid \mathcal{F}_{t}^{\hat{y}}\right] \beta_{t} d t
\end{aligned}
$$

where $\mathcal{F}_{t}^{\hat{y}}=\sigma\left(\hat{y}_{s}, 0 \leq s \leq t\right)$ is the information filtration generated by $\hat{y}$. It is obvious that we can assume that

$$
\mathbb{E}(\tilde{v})=0 \quad \text { and } \quad \mathbb{E}\left(\tilde{v}^{2}\right)=1 .
$$

We shall show that $\tilde{y}$ solves (2.5). This follows from the following lemma.

Lemma 2.1. Assume that

$$
s \rightarrow \frac{\beta_{s}}{\sigma_{s}} \in \mathcal{C}^{2}[0, t]
$$

for all $t<T$. Then $\mathcal{F}_{t}^{\tilde{y}}=\mathcal{F}_{t}^{\hat{y}}$ for all $t \in[0, T]$. 
Proof. Since

$$
d \tilde{y}_{t}=d \hat{y}_{t}-\mathbb{E}\left[\tilde{v} \mid \mathcal{F}_{t}^{\hat{y}}\right] \beta_{t} d t
$$

we see that $\mathcal{F}_{t}^{\tilde{y}} \subset \mathcal{F}_{t}^{\hat{y}}$. We need to prove the other inclusion $\mathcal{F}_{t}^{\hat{y}} \subset \mathcal{F}_{t}^{\tilde{y}}$. First we shall compute $p_{t}:=\mathbb{E}\left[\tilde{v} \mid \mathcal{F}_{t}^{\hat{y}}\right]$ by using the result obtained in [5]. Define

$$
K_{H}(t, s)=\kappa_{H}^{-1} s^{\frac{1}{2}-H}(t-s)^{\frac{1}{2}-H},
$$

where $\kappa_{H}=2 H \Gamma(3 / 2-H) \Gamma(1 / 2+H)$. Let

$$
y_{t}^{*}=\int_{0}^{t} K_{H}(t, s) \sigma_{s}^{-1} d \hat{y}_{s} .
$$

Then from Theorem 1 of [5], we know that $y_{t}^{*}, 0 \leq t \leq T$, is a semimartingale and the information filtrations generated by $y^{*}$ and $\hat{y}$ are the same:

$$
\mathcal{F}_{t}^{y^{*}}=\mathcal{F}_{t}^{\hat{y}}, \quad \forall t \in[0, T] .
$$

We also have

$$
d\left\langle y^{*}, y^{*}\right\rangle_{s}=d \ell(s), \quad d \ell(s):=(2-2 H) \kappa_{H}^{-1} s^{1-2 H} d s
$$

Put

$$
\gamma_{t}=\mathbb{E}\left[\left(\tilde{v}-\mathbb{E}\left[\tilde{v} \mid \mathcal{F}_{t}^{\hat{y}}\right]\right)^{2}\right], \quad t \in[0, T]
$$

and define (which is $p(s, 0)$ of $(13)$ in [5])

$$
\rho_{s}=\rho_{s}(\beta)=\frac{d}{d \ell(s)} \int_{0}^{s} K_{H}(s, r) \frac{\beta_{r}}{\sigma_{r}} d r \quad s \in[0, T]
$$

Then by Section 5.1 of [5] we have

$$
\gamma_{t}=\gamma_{t}(\beta)=\left(\gamma_{0}^{-1}+\int_{0}^{t} \rho_{s}^{2} d \ell(s)\right)^{-1}, \quad t \in[0, T],
$$

for some constant $\gamma_{0} \neq 0$ and

$$
p_{t}=\gamma_{t} \gamma_{0}^{-1}\left(p_{0}+\gamma_{0} \int_{0}^{t} \rho_{s} d y_{s}^{*}\right), \quad \forall t \in[0, T] .
$$

From the definition of $p_{t}$ we have $p_{0}=\mathbb{E}\left[\tilde{v} \mid \mathcal{F}_{0}\right]=\mathbb{E}(\tilde{v})=0$ since we assume $\mathbb{E}(\tilde{v})=0$. Thus we have

$$
p_{t}=\gamma_{t} \int_{0}^{t} \rho_{s} d y_{s}^{*}, \quad \forall t \in[0, T]
$$


From Lemma 5.3, we have

$$
p_{t}=\int_{0}^{t} g(t, s) d \hat{y}_{s}
$$

where

$$
g(t, s)=\gamma_{t}\left[K_{H}(t, s) \rho_{t}-\int_{s}^{t} K_{H}(r, s) \rho_{r}^{\prime} d r\right] \sigma_{s}^{-1}
$$

For any smooth deterministic function $f_{t}, t \in[0, T)$, we now consider

$$
\begin{aligned}
\int_{0}^{t} f_{s} d \tilde{y}_{s} & =\int_{0}^{t} f_{s}\left(d \hat{y}_{s}-\mathbb{E}\left[\tilde{v} \mid \mathcal{F}_{s}^{\hat{y}}\right] \beta_{s} d s\right) \\
& =\int_{0}^{t} f_{s}\left(d \hat{y}_{s}-p_{s} \beta_{s} d s\right) \\
& =\int_{0}^{t} f_{s} d \hat{y}_{s}-\int_{0}^{t} f_{s} \beta_{s} p_{s} d s \\
& =\int_{0}^{t} f_{s} d \hat{y}_{s}-\int_{0}^{t} f_{s} \beta_{s}\left(\int_{0}^{s} g(s, u) d \hat{y}_{u}\right) d s \\
& =\int_{0}^{t}\left(f_{u}-\int_{u}^{t} g(s, u) f_{s} \beta_{s} d s\right) d \hat{y}_{u} .
\end{aligned}
$$

where we have used the Fubini type theorem in (2.20). We want to find a representation of $\hat{y}$ in terms of $\tilde{y}$. This is equivalent to find a solution of the equation

$$
f_{u}-\int_{u}^{t} g(s, u) f_{s} \beta_{s} d s=\chi_{[0, t]}(u) .
$$

By classical results on Volterra equations, see e.g. [4], Lemma 4.3.3 on page 125, this equation has a solution if

$$
\int_{0}^{t} \int_{s}^{t} \beta_{s}^{2} g^{2}(s, r) d r d s<\infty \quad \text { for all } t<T
$$

where $g(s, r)$ is given by (2.19). By the Lemma 5.2 in the Appendix we obtain that if (2.9) holds, then (2.22) is satisfied. Therefore we see that $\mathcal{F}_{t}^{\hat{y}} \subset \mathcal{F}_{t}^{\tilde{y}}$. This concludes the proof of the lemma.

Corollary 2.2. Assume that (2.9) holds. Then $\tilde{y}_{t}, 0 \leq t \leq T$, defined by (2.8) is a solution of $(2.5)$.

Remark 2.3. In view of Corollary 2.2 we choose to represent the total traded volume process $y$ by $\tilde{y}$, and we write $y$ instead of $\tilde{y}$ from now on. Note however, that we have not proved that the solution of (2.5) is unique, so this choice is not totally justified from a mathematical point 
of view, since there might be solutions y of (2.5) which are not representable as innovation processes of linear filtering problems.

On the other hand, since the market makers are assumed to know $\mathcal{F}_{t}^{y}$ and also of course the price $p_{t}$ at any time $t \in[0, T]$, then by (2.3) and (2.7) they know

$$
\hat{y}_{t}=y_{t}+\int_{0}^{t} p_{s} \beta_{s} d s
$$

This implies that

$$
\mathcal{F}_{t}^{\hat{y}}=\mathcal{F}_{t}^{y}
$$

and hence $d y_{t}=d \tilde{y}_{t}$, even without hypothesis (2.9). So from a modeling point of view the assumption that $y_{t}=\tilde{y}_{t}$ is natural, and we will base our study on this.

As shown in the introduction the expected terminal wealth of the insider can be expressed as follows:

$$
\mathbb{E}\left(w_{T}\right)=w_{0}+\int_{0}^{T} \mathbb{E}\left[\left(\tilde{v}-p_{t}\right)^{2}\right] \beta_{t} d t-\int_{0}^{T} \mathbb{E}\left[\left(\tilde{v}-p_{T}\right)\left(\tilde{v}-p_{t}\right)\right] \beta_{t} d t
$$

We need to compute $\mathbb{E}\left[\left(\tilde{v}-p_{T}\right)\left(\tilde{v}-p_{t}\right)\right]$. We have

$$
\begin{aligned}
\mathbb{E}\left[\left(\tilde{v}-p_{T}\right)\left(\tilde{v}-p_{t}\right)\right] & =\mathbb{E}\left(\tilde{v}^{2}\right)-\mathbb{E}\left(\tilde{v} p_{t}\right)-\mathbb{E}\left(\tilde{v} p_{T}\right)+\mathbb{E}\left(p_{T} p_{t}\right) \\
& =\mathbb{E}\left(\tilde{v}^{2}\right)-\mathbb{E}\left(p_{t}^{2}\right)-\mathbb{E}\left(p_{T}^{2}\right)+\mathbb{E}\left(p_{T} p_{t}\right) .
\end{aligned}
$$

We first compute $\mathbb{E}\left(p_{T} p_{t}\right)$. By (1.3) we have that $p_{t}, 0 \leq t \leq T$, is a square-integrable martingale. Hence

$$
\mathbb{E}\left[p_{t} p_{T}\right]=\mathbb{E}\left[p_{t}^{2}\right],
$$

and consequently

$$
\begin{aligned}
\mathbb{E}\left[\left(\tilde{v}-p_{T}\right)\left(\tilde{v}-p_{t}\right)\right] & =\mathbb{E}\left(\tilde{v}^{2}\right)-\mathbb{E}\left(p_{t}^{2}\right)-\mathbb{E}\left(p_{T}^{2}\right)+\mathbb{E}\left(p_{T} p_{t}\right) \\
& =\mathbb{E}\left(\tilde{v}^{2}\right)-\mathbb{E}\left(p_{t}^{2}\right)-\mathbb{E}\left(p_{T}^{2}\right)+\mathbb{E}\left(p_{t}^{2}\right) \\
& =\mathbb{E}\left(\tilde{v}^{2}\right)-\mathbb{E}\left(p_{T}^{2}\right) .
\end{aligned}
$$

But

$$
\mathbb{E}\left(p_{T}^{2}\right)=\mathbb{E}\left(\tilde{v}^{2}\right)-\mathbb{E}\left(\tilde{v}-p_{T}\right)^{2}=\mathbb{E}\left(\tilde{v}^{2}\right)-\gamma_{T},
$$

and

$$
\mathbb{E}\left[\left(\tilde{v}-p_{T}\right)\left(\tilde{v}-p_{t}\right)\right]=\gamma_{T}
$$


Hence by (2.25) and by (2.26) we want to maximize

$$
J(\beta(\cdot))=w_{0}+\int_{0}^{T}\left(\gamma_{t}(\beta)-\gamma_{T}(\beta)\right) \beta_{t} d t .
$$

First let us maximize

$$
J_{0}(\beta(\cdot))=\int_{0}^{T} \gamma_{t}(\beta) \beta_{t} d t
$$

We do this by using a perturbation argument, as in [1]. Let $\varepsilon$ be an arbitrary small number and $\xi_{t}, 0 \leq t \leq T$, be an arbitrary smooth function. We want to compute $\left.\frac{d}{d \varepsilon}\right|_{\varepsilon=0} J_{0}(\beta+\varepsilon \xi)$. In the following we assume that all functions involved are smooth enough to exchange the order of derivation and integration. We first note that by the definition (2.15) of $\rho$ we obtain

$$
\left.\frac{d}{d \varepsilon}\right|_{\varepsilon=0} \rho_{s}(\beta+\varepsilon \xi)=\frac{d}{d \ell(s)} \int_{0}^{s} K_{H}(s, r) \frac{\xi_{r}}{\sigma_{r}} d r
$$

Thus

$$
\begin{aligned}
\left.\frac{d}{d \varepsilon}\right|_{\varepsilon=0} \int_{0}^{t}\left[\rho_{s}(\beta+\varepsilon \xi)\right]^{2} d \ell(s) & =2 \int_{0}^{t} \rho_{s} \frac{d}{d \ell(s)} \int_{0}^{s} K_{H}(s, r) \frac{\xi_{r}}{\sigma_{r}} d r d \ell(s) \\
& =2 \int_{0}^{t} \rho_{s} \frac{d}{d s} \int_{0}^{s} K_{H}(s, r) \frac{\xi_{r}}{\sigma_{r}} d r d s .
\end{aligned}
$$

We apply this result to compute $\left.\frac{d}{d \varepsilon}\right|_{\varepsilon=0} \gamma_{t}(\beta+\varepsilon \xi)$. By (2.16) and (2.29) we have

$$
\begin{aligned}
\left.\frac{d}{d \varepsilon}\right|_{\varepsilon=0} \gamma_{t}(\beta+\varepsilon \xi) & =-2 \gamma_{t}^{2} \int_{0}^{t} \rho_{s} \frac{d}{d s} \int_{0}^{s} K_{H}(s, r) \frac{\xi_{r}}{\sigma_{r}} d r d s \\
& =-2 \gamma_{t}^{2} \rho_{t} \int_{0}^{t} K_{H}(t, r) \frac{\xi_{r}}{\sigma_{r}} d r+2 \gamma_{t}^{2} \int_{0}^{t} \rho_{s}^{\prime} \int_{0}^{s} K_{H}(s, r) \frac{\xi_{r}}{\sigma_{r}} d r d s \\
& =2 \gamma_{t}^{2} \int_{0}^{t} \sigma_{r}^{-1}\left[\int_{r}^{t} \rho_{s}^{\prime} K_{H}(s, r) d s-\rho_{t} K_{H}(t, r)\right] \xi_{r} d r .
\end{aligned}
$$

Putting everything together we obtain

$$
\begin{aligned}
\left.\frac{d}{d \varepsilon}\right|_{\varepsilon=0} J_{0}(\beta+\varepsilon \xi) & =\int_{0}^{T} \gamma_{t} \xi_{t} d t+2 \int_{0}^{T} \beta_{t} \gamma_{t}^{2}\left\{\int_{0}^{t} \sigma_{r}^{-1}\left[\int_{r}^{t} \rho_{s}^{\prime} K_{H}(s, r) d s-\rho_{t} K_{H}(t, r)\right] \xi_{r} d r\right\} d t \\
& =\int_{0}^{T} \gamma_{r} \xi_{r} d r+2 \int_{0}^{T} \sigma_{r}^{-1}\left\{\int_{r}^{T} \beta_{t} \gamma_{t}^{2}\left[\int_{r}^{t} \rho_{s}^{\prime} K_{H}(s, r) d s-\rho_{t} K_{H}(t, r)\right] d t\right\} \xi_{r} d r .
\end{aligned}
$$

Since $\xi_{r}$ is arbitrary, we have

$$
\gamma_{r}=-2 \sigma_{r}^{-1} \int_{r}^{T} \beta_{t} \gamma_{t}^{2}\left[\int_{r}^{t} \rho_{s}^{\prime} K_{H}(s, r) d s-\rho_{t} K_{H}(t, r)\right] d t
$$


or equivalently

$$
\sigma_{r} \gamma_{r}(\beta)=-2 \int_{r}^{T} \beta_{t} \gamma_{t}^{2}(\beta)\left[\int_{r}^{t} \rho_{s}^{\prime}(\beta) K_{H}(s, r) d s-\rho_{t}(\beta) K_{H}(t, r)\right] d t
$$

for $0 \leq t \leq T$. Thus we have proved that if

$$
\beta \rightarrow J_{0}(\beta(\cdot))=\int_{0}^{T} \gamma_{t}(\beta) \beta_{t} d t
$$

is maximal, then $\gamma_{t}(\beta)$ satisfies (2.31). In particular, $\gamma_{T}(\beta)=0$. But this implies that $\gamma_{t}(\beta)$ is also optimal for

$$
J(\beta(\cdot))=w_{0}+J_{0}(\beta(\cdot))=w_{0}+\int_{0}^{T}\left(\gamma_{t}(\beta)-\gamma_{T}(\beta)\right) \beta_{t} d t
$$

since we always have $\gamma_{T}(\beta) \geq 0$ and $\beta \geq 0$. We have proved

Theorem 2.4. Suppose $\beta$ is an optimal insider portfolio for the problem

$$
\sup _{\beta} \mathbb{E}\left[w_{T}(\beta)\right]=\sup _{\beta}\left\{w_{0}+\int_{0}^{T}\left(\gamma_{t}(\beta)-\gamma_{T}(\beta)\right) \beta_{t} d t\right\}
$$

Then $\gamma_{T}(\beta)=0$ and $\gamma_{t}(\beta)$ satisfies equation (2.31). In particular, by (2.26)

$$
p_{T}=\tilde{v}
$$

Proposition 2.5. The process $y$ defined by

$$
d y_{t}=\left(\tilde{v}-\mathbb{E}\left[\tilde{v} \mid \mathcal{F}_{t}^{\hat{y}}\right]\right) \beta_{t} d t+\sigma_{t} d B_{t}^{H}, \quad y_{0}=0
$$

is an $\mathcal{H}_{t}:=\sigma\left(\tilde{v}, B_{s}^{H} ; s \leq t\right)$-adapted solution of the equation

$$
d \tilde{y}_{t}=\left(\tilde{v}-\mathbb{E}\left[\tilde{v} \mid \mathcal{F}_{t}^{\tilde{y}}\right]\right) \beta_{t} d t+\sigma_{t} d B_{t}^{H}, \quad \tilde{y}_{0}=0 .
$$

Proof. That $y$ defined by (2.36) is a solution of equation (2.37) follows from Lemma 2.1.

We now let $H \rightarrow \frac{1}{2}$ in equation (2.30) and show how it converges to the equation for the optimal $\gamma$ in the case $H=\frac{1}{2}$.

Proposition 2.6. For $H \rightarrow \frac{1}{2}$ equation (2.30) becomes

$$
\gamma_{r}=2 \frac{\beta_{r}}{\sigma_{r}^{2}} \int_{r}^{T} \beta_{t} \gamma_{t}^{2} d t, \quad 0 \leq r \leq T,
$$

that is equivalent to the equation (4.28) of [1]:

$$
1=2 \frac{\beta_{r} \gamma_{r}}{\sigma_{r}^{2}} \int_{r}^{T} \beta_{t} \exp \left(-2 \int_{r}^{t} \frac{\gamma_{u} \beta_{u}^{2}}{\sigma_{u}^{2}} d u\right) d t, \quad 0 \leq r \leq T,
$$

for the optimal $\gamma$ in the case $H=\frac{1}{2}$. 
Proof. First of all we note that by $(2.10) K_{H}(s, r)$ converges to 1 for $H \rightarrow \frac{1}{2}$. Furthermore by taking the limit in $(2.15)$ we obtain that $\rho_{t}$ goes to $\frac{\beta_{t}}{\sigma_{t}}$ for all $t \in[0, T]$. Hence $(2.30)$ becomes

$$
\gamma_{r}=2 \sigma_{r}^{-1} \rho_{r} \int_{r}^{T} \beta_{t} \gamma_{t}^{2} d t=2 \frac{\beta_{r}}{\sigma_{r}^{2}} \int_{r}^{T} \beta_{t} \gamma_{t}^{2} d t, 0 \leq r \leq T,
$$

if $H \rightarrow \frac{1}{2}$ by uniform integrability. When $H \rightarrow 1 / 2$, the equation (2.16) becomes

$$
\gamma_{t}=\left(\frac{1}{\gamma_{0}}+\int_{0}^{t}\left(\frac{\beta_{s}}{\sigma_{s}}\right)^{2} d s\right)^{-1}, 0 \leq r \leq T .
$$

Therefore, $\gamma$ satisfies

$$
\frac{d \gamma_{t}}{d t}=-\frac{\beta_{t}^{2}}{\sigma_{t}^{2}} \gamma_{t}^{2}
$$

Hence for $t>r$ we have that

$$
\gamma_{t}=\gamma_{r} \exp \left(-\int_{r}^{t} \frac{\beta_{u}^{2}}{\sigma_{u}^{2}} \gamma_{u} d u\right)
$$

Substituting (2.41) into (2.39), we obtain

$$
\gamma_{r}=2 \frac{\beta_{r}}{\sigma_{r}^{2}} \int_{r}^{T} \gamma_{r}^{2} \beta_{t} \exp \left(-2 \int_{r}^{t} \frac{\gamma_{u} \beta_{u}^{2}}{\sigma_{u}^{2}} d u\right) d t, \quad r \in[0, T]
$$

This is equation (2.38).

\section{Uniqueness of the equation}

The equation (2.5) which we reproduce here

$$
d y_{t}=\left(\tilde{v}-\mathbb{E}\left[\tilde{v} \mid \mathcal{F}_{t}^{y}\right]\right) \beta_{t} d t+\sigma_{t} d B_{t}^{H}, \quad t \in[0, T],
$$

with $y_{0}=0$ is a new type of equation even in the case $B^{H}$ is replaced by a Brownian motion, where $B^{H}$ is a fractional Brownian motion of Hurst parameter $H, \beta_{t}$ and $\sigma_{t}$ are deterministic functions and $\tilde{v}$ is a standard normal random variable independent of the fractional Brownian motion $B^{H}$.

Lemma 2.1 yields the existence of a solution. In Remark 2.3, we explain from economic point of view the rationale of uniqueness. However, mathematically the uniqueness is still an open problem mathematically. It is our conjecture that the uniqueness holds as well.

Here we give an attempt to this problem. We restrict the solution to the form

$$
y_{t}=h_{1}(t) \tilde{v}+\int_{0}^{t} h_{2}(t, s) d B_{s}^{H}
$$


for some unknown functions $h_{1}(t)$ and $h_{2}(t, s), 0 \leq s \leq t \leq T$. Since $\tilde{v}$ and $y_{t}, 0 \leq t \leq T$, are jointly Gaussian, there is a $g(t, s), 0 \leq s \leq t \leq T$, such that (because $\mathbb{E}(\tilde{v})=0$ and $\left.\mathbb{E}(\tilde{v})^{2}=1\right)$

$$
\begin{aligned}
\mathbb{E}\left[\tilde{v} \mid \mathcal{F}_{t}^{y}\right] & =\int_{0}^{t} g(t, s) d y_{s} \\
& =\int_{0}^{t} g(t, s) h_{1}^{\prime}(s) d s \tilde{v}+\int_{0}^{t} g(t, s) h_{2}(s, s) d B_{s}^{H}+\int_{0}^{t} g(t, s) \int_{0}^{s} \frac{\partial}{\partial s} h_{2}(s, r) d B_{r}^{H} d s \\
& =\int_{0}^{t} g(t, s) h_{1}^{\prime}(s) d s \tilde{v}+\int_{0}^{t}\left[g(t, s) h_{2}(s, s)+\int_{s}^{t} g(t, r) \frac{\partial}{\partial r} h_{2}(r, s) d r\right] d B_{s}^{H} .
\end{aligned}
$$

By the property of conditional expectation we have

$$
\mathbb{E}\left(y_{s} \tilde{v}\right)=\mathbb{E}\left(y_{s} \mathbb{E}\left[\tilde{v} \mid \mathcal{F}_{t}^{y}\right]\right), \quad \forall 0 \leq s \leq t .
$$

First we have

$$
\mathbb{E}\left(y_{s} \tilde{v}\right)=h_{1}(s) .
$$

On the other hand, we have

$\mathbb{E}\left(y_{s} \mathbb{E}\left[\tilde{v} \mid \mathcal{F}_{t}^{y}\right]\right)=h_{1}(s) \int_{0}^{t} g(t, u) h_{1}^{\prime}(u) d u$

$$
+\int_{0}^{r} \int_{0}^{t} h_{2}\left(r, s_{1}\right)\left[g\left(t, s_{2}\right) h_{2}\left(s_{2}, s_{2}\right)+\int_{s_{2}}^{t} g(t, r) h_{2}\left(r, s_{2}\right) d r\right] \phi\left(s_{1}-s_{2}\right) d s_{1} d s_{2},
$$

where

$$
\phi(u)=H(2 H-1)|u|^{2 H-2} .
$$

Thus equation (3.4) becomes

$$
\begin{aligned}
h_{1}(s)= & \left.h_{1}(s) \int_{0}^{t} g(t, u) h_{1}^{\prime}(u) d u\right) \\
& +\int_{0}^{r} \int_{0}^{t} h_{2}\left(r, s_{1}\left[g\left(t, s_{2}\right) h_{2}\left(s_{2}, s_{2}\right)+\int_{s_{2}}^{t} g(t, r) h_{2}\left(r, s_{2}\right) d r\right] \phi\left(s_{1}-s_{2}\right) d s_{1} d s_{2},\right.
\end{aligned}
$$

Substituting (3.3) into (3.1), we have

$$
\begin{aligned}
y_{t}= & \int_{0}^{t}\left(\tilde{v}-\mathbb{E}\left[\tilde{v} \mid \mathcal{F}_{r}^{y}\right]\right) \beta_{r} d r+\int_{0}^{t} \sigma_{r} d B_{r}^{H} \\
= & \int_{0}^{t}\left\{\tilde{v}-\tilde{v} \int_{0}^{r} g(r, s) h_{1}^{\prime}(s) d s\right. \\
& \left.+\int_{0}^{r}\left[g(r, s) h_{2}(s, s)+\int_{s}^{r} g(r, u) \frac{\partial}{\partial u} h_{2}(u, s) d u\right] d B_{s}^{H}\right\} \beta_{r} d r+\int_{0}^{t} \sigma_{r} d B_{r}^{H} \\
= & \int_{0}^{t}\left[1-\int_{0}^{r} g(r, s) h_{1}^{\prime}(s) d s\right] \beta_{r} d r \tilde{v} \\
& -\int_{0}^{t}\left\{\int_{s}^{t}\left[g(r, s) h_{2}(s, s)+\int_{s}^{r} g(r, u) \frac{\partial}{\partial u} h_{2}(u, s) d u\right] \beta_{r} d r\right\} d B_{s}^{H}+\int_{0}^{t} \sigma_{r} d B_{r}^{H} .
\end{aligned}
$$


Comparing (3.7) with (3.2) and using the fact that $\tilde{v}$ and $B_{t}^{H}$ are independent, we have

$$
\begin{aligned}
h_{1}(t) & =\int_{0}^{t}\left[1-\int_{0}^{r} g(r, s) h_{1}^{\prime}(s) d s\right] \beta_{r} d r \\
h_{2}(t, s) & =\sigma_{s}-\int_{0}^{t}\left\{\int_{s}^{t}\left[g(r, s) h_{2}(s, s)+\int_{s}^{r} g(r, u) \frac{\partial}{\partial u} h_{2}(u, s) d u\right] \beta_{r} d r\right\} .
\end{aligned}
$$

Thus we obtain

Proposition 3.1. The equation (3.1) has a unique solution of the form (3.2) if the following system of equations has a unique solution $\left(h_{1}(t), h_{2}(t, s), g(t, s), 0 \leq s \leq t \leq T\right)$ :

$$
\left\{\begin{aligned}
h_{1}(t)= & \int_{0}^{t}\left[1-\int_{0}^{r} g(r, s) h_{1}^{\prime}(s) d s\right] \beta_{r} d r \\
h_{2}(t, s)= & \sigma_{s}-\int_{0}^{t}\left\{\int_{s}^{t}\left[g(r, s) h_{2}(s, s)+\int_{s}^{r} g(r, u) \frac{\partial}{\partial u} h_{2}(u, s) d u\right] \beta_{r} d r\right\} \\
h_{1}(s)= & h_{1}(s) \int_{0}^{t} g(t, u) h_{1}^{\prime}(u) d u \\
& +\int_{0}^{r} \int_{0}^{t} h_{2}\left(r, s_{1}\right)\left[g\left(t, s_{2}\right) h_{2}\left(s_{2}, s_{2}\right)+\int_{s_{2}}^{t} g(t, r) h_{2}\left(r, s_{2}\right) d r\right] \phi\left(s_{1}-s_{2}\right) d s_{1} d s_{2} .
\end{aligned}\right.
$$

The existence of the above system was obtained in Section 2 through the technique of filtering.

\section{The impact of memory (persistence) in the noise trades}

One of the motivations of this paper is to investigate how the memory (persistence) and regularity of the noise process of the noise traders, represented by the Hurst coefficient $H>\frac{1}{2}$, influence the performance of the insider.

Unfortunately, we are not able to solve our general equation (2.31) to obtain the optimal $\beta_{t}=\beta_{t}(H), t \in[0, T]$, explicitly, and thus we are unable to make any conclusion about this influence in general. However, if we restrict ourselves to constant insider trading intensity $\beta=\beta(H)>0$, our equations simplify as follows.

Consider $\sigma$ constant. By (5.1) in Lemma 5.1 we obtain that

$$
\begin{aligned}
\rho_{t} & =s^{2 H-2} \frac{\beta}{\sigma} \int_{0}^{s} r^{\frac{1}{2}-H}(s-r)^{\frac{1}{2}-H} d r \\
& =\frac{\beta}{\sigma} \int_{0}^{1} u^{\frac{1}{2}-H}(1-u)^{\frac{1}{2}-H} d u \\
& =\frac{\Gamma\left(\frac{3}{2}-H\right)^{2}}{\Gamma(3-2 H)} \frac{\beta}{\sigma} .
\end{aligned}
$$

Therefore equation (2.16) becomes 


$$
\begin{aligned}
\gamma_{t} & =\left(\gamma_{0}^{-1}+\int_{0}^{t} \rho_{s}^{2} d \ell(s)\right)^{-1} \\
& =\left(\gamma_{0}^{-1}+a_{H} t^{2-2 H} \frac{\beta^{2}}{\sigma^{2}}\right)^{-1},
\end{aligned}
$$

where $a_{H}:=\frac{\Gamma\left(\frac{3}{2}-H\right)^{3}}{2 H \Gamma(3-2 H)^{2} \Gamma\left(\frac{1}{2}+H\right)}$. Hence, we can write down the performance functional as

$$
\begin{aligned}
J(\beta) & =\beta \int_{0}^{T}\left(\gamma_{t}-\gamma_{T}\right) d t \\
& =\beta \int_{0}^{T} \frac{1}{\gamma_{0}^{-1}+a_{H} t^{2-2 H} \frac{\beta^{2}}{\sigma^{2}}} d t-\frac{\beta T}{\gamma_{0}^{-1}+a_{H} T^{2-2 H} \frac{\beta^{2}}{\sigma^{2}}} .
\end{aligned}
$$

It is easy to see that for a given $H \in\left(\frac{1}{2}, 1\right)$ and $T>0, J(\beta)$ is a continuous function of $\beta$, $J(0)=0$ and $\lim _{\beta \rightarrow \infty} J(\beta)=0$. Thus $J(\beta)$ attains its maximum values over all $\beta>0$. We illustrate the relation between $\beta$ and $J(\beta)$ numerically as plots. We choose $T=2, \sigma=1$ and plot the function $J(\beta)$ for the Hurst parameters $H=0.5$ (top curve), $H=0.6$ (second from top), $H=0.75$ (third from top), $H=0.9$ (fourth from top), and $H=1$ (bottom straight line).

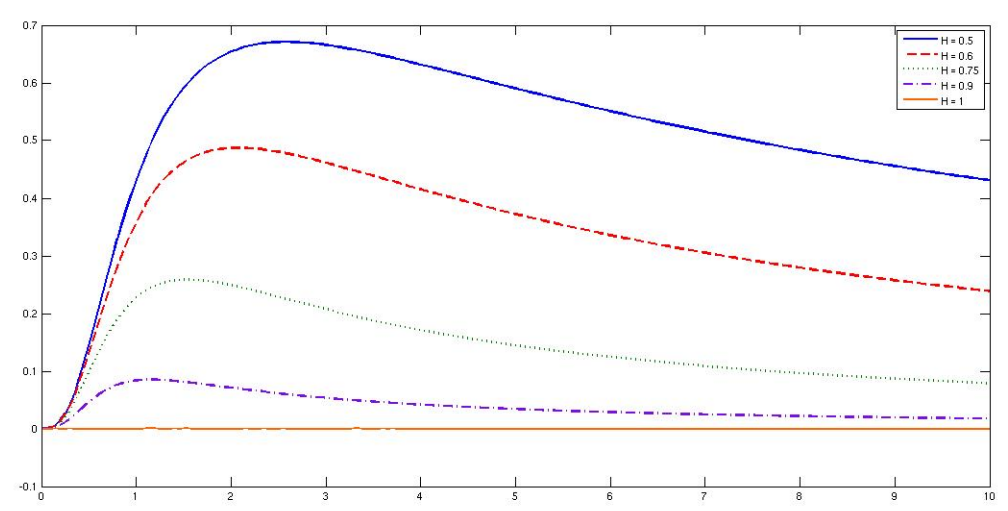

Figure 1: Plot of the functions $J(\beta)$ for 5 different values of $\mathrm{H}$ $\mathrm{x}$-axis is $\beta$ and $\mathrm{y}$-axis is $J(\beta)$

The graphs show that the performance of an insider decreases with increasing $H \in\left[\frac{1}{2}, 1\right)$. They also show that the optimal insider trading intensity $\beta^{*}(H)$ decreases with increasing $H \in\left[\frac{1}{2}, 1\right)$. These results can perhaps be understood as follows. Increasing the Hurst coefficient $H$ of the noise trading reduces the "complexity" of the noise in two ways:

(i) the noise process becomes more persistent, and 
(ii) the paths of the noise process become more regular.

Both these effects contribute to the decrease of the information advantage of the insider, because with reduced noise the actions of the insider become more apparent to the market makers. Thus increasing $H$ might have the same effect on the insider performance as reducing the noise level $|\sigma|$ in the classical Brownian motion model $\left(H=\frac{1}{2}\right)$.

Similarly, the decrease of the optimal trading intensity $\beta^{*}(H)$ with increasing $H$, is also in line with what happens when $|\sigma|$ decreases in the classical setting. (See Section 1).

It is not clear, though, what the effects of increasing $H$ would be if the portfolios $\beta$ were allowed to vary with time. Then the insider might be able to take advantage of the increased "predictability" of the noise traders to increase her performance, and this might outweigh the disadvantage coming from reduced noise complexity mentioned above.

In either case, we have not been able to give rigorous proofs of any of these statements regarding the effects of increasing $H$, and we leave the task of doing so as an open problem.

\section{Appendix}

In this appendix we provided some technical computations needed in the previous sections.

Lemma 5.1. If $\beta_{r}, \sigma_{r}$ are twice differentiable and $\sigma_{r}>0$ on $[0, T]$, then

$$
\begin{gathered}
\rho_{s}=s^{2 H-2} \int_{0}^{s} r^{\frac{1}{2}-H}(s-r)^{\frac{1}{2}-H} \frac{\beta_{r}}{\sigma_{r}} d r \\
+(2-2 H)^{-1} s^{2 H-2} \int_{0}^{s} r^{\frac{3}{2}-H}(s-r)^{\frac{1}{2}-H} \frac{d}{d r}\left[\frac{\beta_{r}}{\sigma_{r}}\right] d r \\
\rho_{s}^{\prime}=-\left[1+(2-2 H)^{-1}\right] s^{2 H-3} \int_{0}^{s} r^{\frac{3}{2}-H}(s-r)^{\frac{1}{2}-H} \frac{d}{d r}\left[\frac{\beta_{r}}{\sigma_{r}}\right] d r \\
-(2-2 H)^{-1} s^{2 H-3} \int_{0}^{s} r^{\frac{5}{2}-H}(s-r)^{\frac{1}{2}-H} \frac{d^{2}}{d r^{2}}\left[\frac{\beta_{r}}{\sigma_{r}}\right] d r
\end{gathered}
$$

for $0 \leq t \leq T$. 
Proof. By definition (2.15) for $\rho$, we obtain

$$
\begin{aligned}
\rho_{s}= & \frac{d}{d \ell(s)} \int_{0}^{s} K_{H}(s, r) \frac{\beta_{r}}{\sigma_{r}} d r \\
= & (2-2 H)^{-1} \kappa_{H} s^{2 H-1} \frac{d}{d s}\left[\int_{0}^{s} K_{H}(s, r) \frac{\beta_{r}}{\sigma_{r}} d r\right] \\
= & (2-2 H)^{-1} s^{2 H-1} \frac{d}{d s}\left[s^{2-2 H} \int_{0}^{1} u^{\frac{1}{2}-H}(1-u)^{\frac{1}{2}-H} \frac{\beta_{s u}}{\sigma_{s u}} d u\right] \\
= & (2-2 H)^{-1} s^{2 H-1}\left[(2-2 H) s^{1-2 H} \int_{0}^{1} u^{\frac{1}{2}-H}(1-u)^{\frac{1}{2}-H} \frac{\beta_{s u}}{\sigma_{s u}} d u\right. \\
& \left.\quad+s^{2-2 H} \int_{0}^{1} u^{\frac{1}{2}-H}(1-u)^{\frac{1}{2}-H} \frac{d}{d s}\left[\frac{\beta_{s u}}{\sigma_{s u}}\right] d u\right] \\
= & \int_{0}^{1} u^{\frac{1}{2}-H}(1-u)^{\frac{1}{2}-H} \frac{\beta_{s u}}{\sigma_{s u}} d u+(2-2 H)^{-1} s \int_{0}^{1} u^{\frac{1}{2}-H}(1-u)^{\frac{1}{2}-H} \frac{d}{d s}\left[\frac{\beta_{s u}}{\sigma_{s u}}\right] d u \\
= & s^{2 H-2} \int_{0}^{s} r^{\frac{1}{2}-H}(s-r)^{\frac{1}{2}-H} \frac{\beta_{r}}{\sigma_{r}} d r \\
& \quad+(2-2 H)^{-1} s^{2 H-2} \int_{0}^{s} r^{\frac{3}{2}-H}(s-r)^{\frac{1}{2}-H} \frac{d}{d r}\left[\frac{\beta_{r}}{\sigma_{r}}\right] d r .
\end{aligned}
$$

Taking the derivative again we have

$$
\begin{aligned}
\rho_{s}^{\prime}= & {\left[1+(2-2 H)^{-1}\right] \int_{0}^{1} u^{\frac{1}{2}-H}(1-u)^{\frac{1}{2}-H} \frac{d}{d s}\left[\frac{\beta_{s u}}{\sigma_{s u}}\right] d u } \\
& +(2-2 H)^{-1} s \int_{0}^{1} u^{\frac{1}{2}-H}(1-u)^{\frac{1}{2}-H} \frac{d^{2}}{d s^{2}}\left[\frac{\beta_{s u}}{\sigma_{s u}}\right] d u
\end{aligned}
$$

Denote $r=s u$. Then

$$
\frac{d}{d s}\left[\frac{\beta_{s u}}{\sigma_{s u}}\right]=u \frac{d}{d r}\left[\frac{\beta_{r}}{\sigma_{r}}\right], \quad \frac{d^{2}}{d s^{2}}\left[\frac{\beta_{s u}}{\sigma_{s u}}\right]=u^{2} \frac{d^{2}}{d r^{2}}\left[\frac{\beta_{r}}{\sigma_{r}}\right] .
$$


Therefore,

$$
\begin{aligned}
\rho_{s}^{\prime}=[1+ & \left.(2-2 H)^{-1}\right] \int_{0}^{1} u^{\frac{1}{2}-H}(1-u)^{\frac{1}{2}-H} \frac{d}{d s}\left[\frac{\beta_{s u}}{\sigma_{s u}}\right] d u \\
& +(2-2 H)^{-1} s \int_{0}^{1} u^{\frac{1}{2}-H}(1-u)^{\frac{1}{2}-H} \frac{d^{2}}{d s^{2}}\left[\frac{\beta_{s u}}{\sigma_{s u}}\right] d u \\
=[1+ & \left.(2-2 H)^{-1}\right] \int_{0}^{1} u^{\frac{3}{2}-H}(1-u)^{\frac{1}{2}-H} \frac{d}{d r}\left[\frac{\beta_{r}}{\sigma_{r}}\right] d u \\
& +(2-2 H)^{-1} s \int_{0}^{1} u^{\frac{5}{2}-H}(1-u)^{\frac{1}{2}-H} \frac{d^{2}}{d r^{2}}\left[\frac{\beta_{r}}{\sigma_{r}}\right] d u \\
=-[1 & \left.+(2-2 H)^{-1}\right] s^{2 H-3} \int_{0}^{s} r^{\frac{3}{2}-H}(s-r)^{\frac{1}{2}-H} \frac{d}{d r}\left[\frac{\beta_{r}}{\sigma_{r}}\right] d r \\
& +(2-2 H)^{-1} s^{2 H-3} \int_{0}^{s} r^{\frac{5}{2}-H}(s-r)^{\frac{1}{2}-H} \frac{d^{2}}{d r^{2}}\left[\frac{\beta_{r}}{\sigma_{r}}\right] d r .
\end{aligned}
$$

Lemma 5.2. Suppose

$$
\sup _{0 \leq r \leq t}\left\{\left|\frac{\beta_{r}}{\sigma_{r}}\right|+\left|\frac{d}{d r}\left(\frac{\beta_{r}}{\sigma_{r}}\right)\right|+\left|\frac{d^{2}}{d r^{2}}\left(\frac{\beta_{r}}{\sigma_{r}}\right)\right|\right\}<\infty .
$$

Then

$$
\sup _{0 \leq s \leq t}\left[\left|\rho_{s}\right|+\left|\rho_{s}^{\prime}\right|\right]<\infty
$$

Proof. We use $C$ to denote a generic constant which may have different value in different occurrences. From Lemma 5.1 and the assumption, we have

$$
\begin{aligned}
\left|\rho_{s}\right| & \leq C\left[s^{2 H-1} \int_{0}^{s} r^{\frac{1}{2}-H}(s-r)^{\frac{1}{2}-H} d r+s^{2 H-2} \int_{0}^{s} r^{\frac{3}{2}-H}(s-r)^{\frac{1}{2}-H} d r\right] \\
& \leq C s \leq C . \\
\left|\rho_{s}^{\prime}\right| & \leq C\left[s^{2 H-3} \int_{0}^{s} r^{\frac{3}{2}-H}(s-r)^{\frac{1}{2}-H} d r+s^{2 H-3} \int_{0}^{s} r^{\frac{5}{2}-H}(s-r)^{\frac{1}{2}-H} d r\right] \\
& \leq C+C s \leq C .
\end{aligned}
$$

Finally we need to express $\int_{0}^{t} \rho_{s} d y_{s}^{*}$ in terms of $\int_{0}^{t} \tilde{\rho}_{s} d \hat{y}_{s}$, where $y^{*}$ and $\hat{y}$ are defined through (2.11).

Introduce the following operator

$$
T(f)(t)=\frac{d}{d t} \int_{0}^{t} K_{H}(t, s) f_{s} d s, \quad f \in C^{1}([0, T] ; \mathbb{R}) .
$$


Then up to an argument of approximation for $y_{t}, 0 \leq t \leq T$, by smooth functions and from

$$
y_{s}^{*}=\int_{0}^{s} K_{H}(s, r) \sigma_{r}^{-1} d \hat{y}_{r}=\int_{0}^{s} K_{H}(s, r) \sigma_{r}^{-1} \dot{\hat{y}}_{r} d r
$$

we can write

$$
\int_{0}^{t} \rho_{s} d y_{s}^{*}=\int_{0}^{t} \rho_{s} T\left(\sigma^{-1} \dot{\hat{y}}\right)(s) d s .
$$

Let $T_{t}^{*}$ be the transpose of $T$ on the interval $[0, t]$, more precisely, $T_{t}^{*}$ is defined by the following identity:

$$
\int_{0}^{t} g_{r}(T(f)(r)) d r=\int_{0}^{t}\left(T_{t}^{*}(g)(r)\right) f_{r} d r, \forall \text { nice smooth functions } \quad f, g \in C^{1}([0, T] ; \mathbb{R}) .
$$

Then

$$
\int_{0}^{t} \rho_{s} d y_{s}^{*}=\int_{0}^{t}\left(T_{t}^{*}(\rho)(s) \sigma_{s}^{-1} d \hat{y}_{s}\right.
$$

A simple computation yields that

$$
T_{t}^{*}(\rho)(s)=K_{H}(t, s) \rho_{t}-\int_{s}^{t} K_{H}(r, s) \rho_{r}^{\prime} d r .
$$

Therefore, we have

Lemma 5.3. For any continuous function $\rho:[0, T] \rightarrow \mathbb{R}$, we have

$$
\int_{0}^{t} \rho_{s} d y_{s}^{*}=\int_{0}^{t}\left[K_{H}(t, s) \rho_{t}-\int_{s}^{t} K_{H}(r, s) \rho_{r}^{\prime} d r\right] \sigma_{s}^{-1} d \hat{y}_{s}
$$

\section{Acknowledgment}

We thank Jan Widenmann for help with the numerical computations and graphs.

1) Department of Mathematics, Ludwig-Maximilians Universität, D-80333 Munich, Germany, email: francesca.biagini@math.lmu.de, meyerbra@math.lmu.de.

2) Department of Mathematics, University of Kansas 405 Snow Hall, Lawrence, Kansas 66045-2142, USA, email: hu@math.ku.edu.

3) Center of Mathematics for Applications (CMA) Department of Mathematics, University of Oslo Box 1053 Blindern, N-0316, Oslo, Norway, email: oksendal@math.uio.no.

4) Norwegian School of Economics and Business Administration, Helleveien 30, N-5045, Bergen, Norway. 


\section{References}

[1] Aase K. K., Bjuland T. and Øksendal B. (2010) Strategic insider trading equilibrium: a filter theory approach. To appear in Afrika Matematica.

[2] Back, K. (1992). Insider trading in continuous time. The Review of Financial Studies 5 (3), 387-409.

[3] Biagini F., Hu Y., Øksendal B., Zhang T. (2008) Stochastic Calculus for Fractional Brownian Motion and Applications, Springer.

[4] Davis, M. H. A. (1977) Linear Estimation and Stochastic Control. Chapman and Hall.

[5] Kleptsyna, M. L., Le Breton, A. and Roubaud, M. C. (2000) General approach to filtering with fractional Brownian noises - application to linear systems, Stochastics and Stochastics Rep. 71, n. 1-2, 119-140.

[6] Kyle, A. S. (1985). Continuous auctions and insider trading. Econometrica 53 (6), 13151336.

[7] Mishura, Y. (2008) Stochastic Calculus for Fractional Brownian Motion and Related Processes, Springer.

[8] Russo, F. and Vallois, P. (2000) Stochastic calculus with respect to continuous finite quadratic variation processes, Stochastics and Stochastics Reports 70, 1-40. 